\title{
GI Dysmotility: A CASE RePort
}

Katie Osley, MD and Yiu Tak Leung, MD, PhD

\section{Case Report}

A 75-year-old male, with a medical history of diabetes, hypertension, coronary artery disease, status post coronary artery bypass graft, and left-sided breast cancer, status post left breast mastectomy, was transferred from an outside hospital with complaints of a month of constipation, nausea and vomiting. The patient presented to an OSH a month prior with recent onset of constipation, with no bowel movements for 10 days, changed from his usual habit of daily bowel movements. He initially responded to lactulose with a bowel movement and was discharged on a regimen of stool softeners and laxatives; however, upon returning home, he was again unable to move his bowels despite his bowel regimen and developed diffuse abdominal pain, nausea and vomiting secondary to distension. An outpatient esophagogastroduodenoscopy (EGD) showed that the patient had a normal esophagus and a large amount of retained food in the stomach, concerning for gastroparesis. This finding was thought to be secondary to his diabetes, despite his well-controlled blood sugars and a hemoglobin A1c of 7.0 $\%$. After two further weeks of constipation, the patient was readmitted to the $\mathrm{OSH}$ with abdominal pain, intractable nausea and vomiting, as well as a 20 -25 lbs weight loss since his symptoms began. Imaging showed colonic gaseous distention, with the cecum dilated to $9.5 \mathrm{~cm}$, and an un-prepped sigmoidoscopy was performed, showing no inflammation, polyps or masses; of note, a screening colonoscopy done 4 months prior identified and removed multiple benign polyps. A CT scan of his abdomen and pelvis also did not show any obstructing colonic masses. He was presumed to have colonic pseudo-obstruction, or Ogilvie Syndrome. Erythromycin was started without effect. He was subsequently given neostigmine, which was also unsuccessful in relieving his symptoms. The patient was then referred to another OSH for further workup. A 4-day gut motility study showed a pan-GI dysmotility disorder. Furthermore, a gastric emptying study revealed markedly delayed gastric emptying of both solid and liquid foods, but defecography was entirely normal. Non-contrast CTs of the chest, abdomen and pelvis again did not identify any masses or bowel obstruction, but did show enlarged precarinal lymph nodes and prostatomegaly, as well as small thyroid nodules bilaterally. As the patient continued to fail to have a bowel movement and could not tolerate a diet, he was started on total parenteral nutrition and was transferred to Thomas Jefferson University Hospital (TJUH) for further evaluation.

On presentation to TJUH, the patient complained of occasional diffuse abdominal pain and distention, as well as nausea. He had not had any vomiting since he stopped eating when he was started on TPN. He denied any swallowing difficulty but did complain of constantly spitting up clear sputum. He had not had a bowel movement in more than 4 weeks since his initial response to lactulose at the first OSH. He had lost $30 \mathrm{lbs}$ in the 4 weeks since his symptoms began. His review of systems was other wise negative.

As noted previously, the patient's medical history is significant for hypertension, hyperlipidemia, coronary artery disease status post coronary artery bypass graft 15 years ago, well-controlled type 2 diabetes diagnosed 8 years ago and benign prostate hypertrophy. He also had left-sided breast cancer 7 years ago, treated with a left mastectomy and a 5-year course of tamoxifen. Social history indicated no current abuse of alcohol, tobacco, or illegal drugs, though he admitted to a 20-pack-year smoking history, having quit 35 years ago. He is a long time employee of a steel company and also owns a farm in eastern Pennsylvania. Family history is significant for breast cancer in his sister and stomach cancer in his mother.

The patient's admission labs were unremarkable, with a normal complete blood count, prothrombin time, partial thromboplastin time, basic metabolic panel and electrolytes. His thyroid function tests were also within normal limits. He did have an elevated prostate specific antigen of $11.6 \mathrm{ng} / \mathrm{mL}$. On physical examination, the patient was afebrile with normal vital signs. He was alert and oriented to person, place and time, and in no distress. Of note, he had post-CABG median sternotomy scar that was well healed, as well as a left-sided scar on his left chest remnant of a mastectomy. His abdomen was soft with active bowel sounds, but diffusely distended and diffusely tender to palpation, without guarding or rebound. The remainder of his exam was otherwise benign.

\section{Hospital Course}

The patient was admitted for workup of his GI dysmotility. Initial imaging with an obstruction series again showed no evidence of an intestinal obstruction. A subsequent CT of the abdomen and pelvis demonstrated a normally appearing small and large bowel and identified no cause of his symptoms. He then underwent an EGD, which found esophagitis at the level of his gastroesophageal junction; however, the remainder of his esophagus appearing normal. His stomach had retained fluid and food in the fundus despite having not eaten for almost 2 weeks. The remainder of his stomach exam was remarkable for a spastic pylorus, which was treated with botox injection. There was no improvement in the patient's gastric motility, however, and his symptoms of nausea, abdominal fullness and constipation persisted.

The patient was seen by the Neurology Service to evaluate the possibility of autonomic dysfunction as the etiology of his GI dysmotility. He was found to have orthostatic hypotension with a supine blood pressure of $182 / 80 \mathrm{mmHg}$ and heart rate of 121 beats per minute and an upright blood pressure of 122/65 mmHg 
with a heart rate in the 180 s beats per minute. Though the patient had previously been given a trial of neostigmine at the OSH, he was empirically started on pyridostigmine $60 \mathrm{mg}$ three times a day. He received 5 days total of pyridostigmine, without relief of his constipation. The medication was promptly stopped on the fifth day when daily monitoring EKG showed development of first-degree heart block and bradycardia. EKGs following the cessation of pyridostigmine showed improvement of his $\mathrm{PR}$ interval and resolution of his bradycardia. Acetylcholine receptor binding antibody was checked and returned as normal. To further evaluate for autonomic GI dysmotility, a ganglionic acetylcholine receptor binding antibody titer was sent, which was also negative.

The Rheumatology Service was consulted regarding the possibility of an autoimmune etiology, such as systemic sclerosis, causing his GI dysmotility. The patient's antinuclear antibody titer was positive, with a titer of 1:320 and a speckled pattern; however, the rest of his rheumatologic serologies, including anti-double stranded DNA, anti-sm, anti-RNP, Sjogren's anti-SCL 70, anti-centromere and rheumatoid factor, were negative. Therefore, it was deemed very unlikely that his GI dysmotility was secondary to an autoimmune process. The possibility of infiltrative diseases, such as amyloidosis, causing his GI dysmotility was also examined; however, his serum and urine protein electrophoreses were both negative. Environmental exposures were also explored, given the patient's social history of living on a farm. Lyme antibody and a heavy metal drug screen were negative.

There was significant suspicion that the patient's GI dysmotility may be secondary to a paraneoplastic syndrome. Accordingly, he was evaluated with a serum paraneoplastic panel. This screen was positive for the anti-Hu (ANNA-1) antibody, which is associated with small cell lung cancer, prostate, and breast cancer. Though CT scans of the patient's chest, abdomen and pelvis were negative for any masses, some enlarged precarinal lymph nodes and an enlarged prostate were noted. Given the patient's history of breast cancer, as well as elevated PSA $(11.6 \mathrm{ng} / \mathrm{mL})$ and a remote smoking history, the patient was recommended to get an outpatient positron emission tomogram (PET) scan to workup any possible occult malignancy. He was also recommended have biopsies done of his precarinal lymph nodes and his prostate, regardless of the PET findings. The patient was started empirically on intravenous steroids, along with lubiprostone to increase GI secretions. Approximately 5 days after being started on methylprednisolone $40 \mathrm{mg}$ daily, and lubiprostone $24 \mathrm{mg}$ twice a day, he was able to move his bowels, with a total of 4 bowel movements that day, suggesting that his symptoms were secondary a paraneoplastic syndrome from an occult malignancy. Though the patient did not continue to have bowel movements on the steroids and was still unable to tolerate a solid diet, he was discharged home with oral steroids and on a full liquids diet in order to facilitate the completion of an outpatient PET scan to screen for the highly-suspected occult malignancy.

\section{Background/Epidemiology}

Intestinal dysmotility has long been recognized as a paraneoplastic syndrome in small cell carcinoma of the lung (Ahmed and Schuffler). In 1991, Lennon et al. first recognized the association of gastrointestinal dysmotility and ANNA- $1 .{ }^{5}$

\section{Clinical Characteristics/Differential Diagnosis/Serological Testing And Other Studies}

The presence of ANNA-1 (or anti-Hu) can produce a variety of different clinical presentations depending on which part of the gastrointestinal tract is affected. Pseudo-achalasia, gastroparesis and intestinal pseudo-obstruction have all been linked to the autoantibody anti-Hu. ${ }^{5,7,13,16,17}$ The autoantibodies found in patients with paraneoplastic GI dysmotility are different from those thought to be responsible for primary achalasia in that the former may affect neurons throughout the GI track. Therefore, many patients have a pan-dysmotility, although usually one of the above disorders will present as the predominant symptom. Gastroparesis is the most common presentation of GI dysmotility secondary to ANNA-1 antibodies and has been reported to resolve completely upon resection of associated tumors. ${ }^{7}$ These patients present with a marked delay of gastric emptying in the absence of gastric outlet obstruction. This produces symptoms of nausea, vomiting, early satiety, epigastric fullness and retained food in the stomach. On the other hand, patients with ANNA-1-induced pseudo-obstruction will present with either recurrent or persistent symptoms of bowel obstruction. Chronic neurogenic intestinal pseudo-obstruction is defined as severe impairment of intestinal motility producing symptoms of mechanical obstruction in the absence of any organic obstacle to intestinal transit. ${ }^{3}$ An obstructive etiology was ruled out in our patient via obstructive series films, CT of the abdomen and pelvis, and colonoscopy.

The diagnosis of paraneoplastic GI dysmotility requires a high index of suspicion. There are many alternative diagnoses that need to be considered during the work-up of achalasia, gastroparesis and chronic pseudo-obstruction. The differential diagnosis of gastrointestinal neuropathies associated with motor dysfunction include developmental, degenerative (idiopathic, paraneoplastic and infectious), toxic or drug induced, or systemic disease related (diabetes mellitus, scleroderma, amyloidosis) neuropathies. Cytomegalovirus, herpes simplex virus and trypanosome cruzi (Chaga's disease) are the infections most often associated with degenerative inflammatory neuropathies. ${ }^{3}$ In our case, an investigation for infectious etiologies was negative including CMV PCR and stool studies. The patient remained afebrile and never had an elevated white blood cell count throughout the hospital course. A heavy metal drug screen came back negative and a thorough review of our patient's medications failed to find a culprit. Negative serum and urine protein electrophoresis studies and a hemoglobin A1c 
of $7 \%$ helped to rule out infiltrative processes and a diabetic autonomic neuropathy, respectively.

Paraneoplastic gastrointestinal dysmotility can be attributed to the recognition of the RNA binding protein, $\mathrm{Hu}$, by anti-neuronal nuclear antibody (ANNA-1). These autoantibodies are thought to target tumor antigens that show cross-reactivity with myenteric neurons. ${ }^{1}$ Small cell lung carcinoma and thymoma are the most common neoplasms associated with paraneoplastic intestinal dysmotility due to ANNA-1 or anti-Hu antibodies. ${ }^{4,5,15}$ However, associaton with lymphomas and tumors of the breast, prostate and ovary have also been reported. ${ }^{7}$

Lee et al. published a case series of patients with paraneoplastic GI motor dysfunction and found that small cell carcinoma of the lung (SCLC) presented an average of 8.7 months after the onset of GI symptoms in ANNA-1 positive individuals. This is opposed to the other associated malignancies (lymphoma, anaplastic lung adenocarcinoma and ovarian cancer) in which GI symptoms began an average of 14 months after the tumor was diagnosed. Of note, the latter individuals were seropositive for PCA-1 or N-type calcium antibodies rather than ANNA-1. In the same study, it was shown that $83 \%$ of the cases involved a second manifestation of a paraneoplastic disorder, including sympathetic and/or parasympathetic failure, peripheral sensorimotor neuropathy, syndrome of inappropriate anti-diuretic hormone, or cerebellar degeneration. ${ }^{6}$ Therefore, the presence of a neurologic disorder combined with a GI dysmotility may lead the clinician to search for underlying malignant causes. Our patient presented with asymptomatic orthostatic hypotension, which provided a clue that led our team to investigate neurologic causes of gastroparesis and pseudo-obstruction.

Breast tumors have rarely been associated with paraneoplastic GI dysmotility. One patient with bilateral breast cancer, which was treated with chemotherapy, achieved improvement of her concomitant gastroparesis as her tumor went into remission. The patient was presumed to have a paraneoplastic cause of her GI dysmotility and Berghmans et al. hypothesized that auto-antibodies were responsible due to the lack of mechanical obstruction and close clinical correlation. ${ }^{2}$ Our patient's past medical history of the breast carcinoma prompted us to wonder if his clinical anti-Hu syndrome was a manifestation of his tumor recurrence. This is further supported by reports that gastrointestinal paresis and intestinal pseudo-obstruction may be the first sign of a recurring thymoma. ${ }^{4}$

\section{Management (Cancer Workup) and Treatment}

In $13 \%$ of patients with SCLC , an unrelated primary malignancy exists, most commonly renal cell carcinoma. ${ }^{7}$ This suggests that a work-up for SCLC should still be pursued despite the presence of an alternate primary tumor. Our patient had a remote history of a breast tumor for which he had a mastectomy and tamoxifen therapy. He also had an elevated PSA and $6.8 \mathrm{~cm}$ prostate on CT scan that led us to consult urology for prostate biopsy. However, the procedure could not be performed secondary to a urinary tract infection that complicated the patient's hospital course. Despite the evidence for other sources of a paraneoplastic tumor, our clinical suspicion for a SCLC remained high.

Kashyap et al. suggests that serial CT of the chest every 6 months is one conservative management strategy if the initial CT is negative. A PET scan with biopsy of suspicious nodes is an alternative approach. Finally, if the clinical picture is highly suggestive of an occult malignancy despite a negative initial work-up, bronchoscopy or mediastinoscopy may be offered to the patient. ${ }^{1}$ In the case of our patient, his initial chest CT was negative except for a few enlarged precarinal lymph nodes. It was decided that a lymph node biopsy would be offered to the patient if the PET scan failed to show a source. PET scan will allow simultaneous investigation for a breast carcinoma, prostate tumor or an occult SCCL. Finally, a full thickness intestinal biopsy may aid in this diagnosis by demonstrating an inflammatory infiltrate of lymphocytes and plasma cells that inhabit the myenteric plexus and lead to destruction of neural elements. ${ }^{3}$ However, a tissue diagnosis is not always necessary when gastrointestinal dysmotility occurs in conjunction with the presence of a tumor and circulating autoantibodies. Therefore, we suggest that serologic and other non-invasive tests take place first before considering this procedure.

There is no standard of care for the treatment of antibodyassociated GI dysmotility, due to the rarity of the disease and the difficulty measuring response to therapy. Many different regimens combining prokinetic agents, systemic steroid therapy and other immunosuppressants have been reported. Pulse doses of neostigmine ( $2 \mathrm{mg}$ administered intravenously) led to peristalsis and relief of colonic distention in a patient with positive ANNA-1 with paraneoplastic encephalomyelitis and autonomic neuropathy. ${ }^{12}$ A steroid taper starting at $60 \mathrm{mg}$ per day of methylprednisolone led to a remission of symptoms for one year in a patient with idiopathic mesenteric ganglionitis. ${ }^{9} \mathrm{~A}$ regimen of pulse steroids using methylprednisolone $100 \mathrm{mg}$ IV for three days improved symptoms of intestinal subocclusion in another patient with positive ANNA-1. ${ }^{11}$ On the other hand, Lucchinetti et al. reported that 38 patients with positive ANNA-1 and gastrointestinal dysmotility failed to respond to adrenal corticosteroids, plasma exchange, intravenous immune globulin (IVIG) or cyclophosphamide. ${ }^{7}$ Our patient had a limited response to neostigmine, pyridostigmine and erythromycin. However, after starting lubiprostone and intravenous steroids our patient did have a bowel movement and was able to tolerate a full liquid diet. He was sent home on a taper of oral steroids, but relapsed a few weeks later, requiring another hospitalization for intractable nausea, vomiting and abdominal pain.

\section{References}

1. Kashyap P, Farrugia G. Enteric Autoantibodies and Gut Motility Disorders. Gastroenterol Clin North Am. 2008 June; 37 (2): 397-410. 
2. Berghmans T, Musch W, Brenez D, et al. Paraneoplastic gastroparesis. Rev Med Brux 1993; 14(9-10): 275-278.

3. De Giorgio R, Stanghellini V, Barbara G, et al. Primary enteric neuropathies underlying gastrointestinal motor dysfunction. Scand J Gastroenterol. 2000 Feb; 35(2):114-22.

4. Simonelli M, Banna GL, Santoro A. Thymoma associated with myasthenia and autonomic anti-Hu paraneoplastic neuropathy. Tumori. 2009 Mar-Apr; 95(2):243-7.

5. Lennon VA, Sas DF, Busk MF, et al. Enteric neuronal autoantibodies in pseudo-obstruction with small-cell lung carcinoma. Gastroenterology. 1991 Jan; $100(1): 137-42$.

6. Lee HR, Lennon VA, Camilleri M, et al. Paraneoplastic gastrointestinal motor dysfunction: clinical and laboratory characteristics. Am J Gastroenterol. 2001 Feb; 96(2):373-9.

7. Lucchinetti CF, Kimmel DW, Lennon VA. Paraneoplastic and oncologic profiles of patients seropositive for type 1 antineuronal nuclear autoantibodies. Neurology 1998; 50(3):652-657.

8. Vernino S, Low PA, Fealey RD, et al. Autoantibodies to ganglionic acetylcholine receptors in autoimmune autonomic neuropathies. N Engl J Med 2000; 343(12):847-855

9. De Giorgio R, Barbara G, Stanghellini V, et al. Idiopathic myenteric ganglionitis underlying intractable vomiting in a young adult. Eur J Gastroenterol Hepatol. 2000 Jun; 12(6):613-6,

10. Smith VV, Gregson N, Foggensteiner L, et al. Acquired intestinal aganglionosis and circulating autoantibodies without neoplasia or other neural involvement.
11. De Girogio R, Barbaara G, Stanghellini V, et al. Clinical and morphofunctional features of idiopathic myenteric ganglionitis underlying severe intestinal motor dysfunction: a study of three cases. Am J Gastroenterol. 2002 Sep; 97(9):2454-9.

12. Calvet X, Martinez JM, Martinez M. Repeated neostigmine dosage as palliative treatment for chronic colonic pseudo-obstruction in a patient with autonomic paraneoplastic neuropathy. Am J Gastroenterol. 2003 Mar; 98(3):708-9.

13. Anderson NE, Hutchinson DO, Nicholson GJ, et al. Intestinal pseudoobstruction, myasthenia gravis, and thymoma. Neurology. 1996 Oct; 47(4):985-7.

14. Pasha SF, Lunsford TN, Lennon VA. Autoimmune gastrointestinal dysmotility treated successfully with pyridostigmine. Gastroenterology 2006; 131(5):15921596.

15. Kiers L, Altermatt JH, Lennon VA. Paraneoplastic anti-neuronal nuclear IgG autoantibiodies (type I) localize antigen in small cell lung carcinoma. May Clin Proc 1991; 66(12):1209-1216.

16. Gockel I, Eckardt VF, Schmitt, et al. Pseudoachalasia: a case series and analysis of the literature. Scand J Gastroenterol 2005; 40(4):378-385. PMID: 16028431.

17. Liu W, Fackler W, Rice TW, et al. The pathogenesis of pseudoachalasia: a clinicopathologic study of 13 cases of a rare entity. Am J Surg Pathol 2002 26(6):784-788. PMID:12023584

18. Lennon VA, Camilleri M, Miller LJ. Enteric neuronal autoantibodies in pseudoobstruction wit small cell lung carcinoma. Gastroenterology. 1991; 101:1143-1144. Letter.

19. Condom E, Vidal A, Rota R, et al. Paraneoplastic intestinal pseudo-obstruction associated with high titers of Hu autoantibodies. Virchows Archiv A, Pathol Anat Histopathol 1993; 423:507-511.

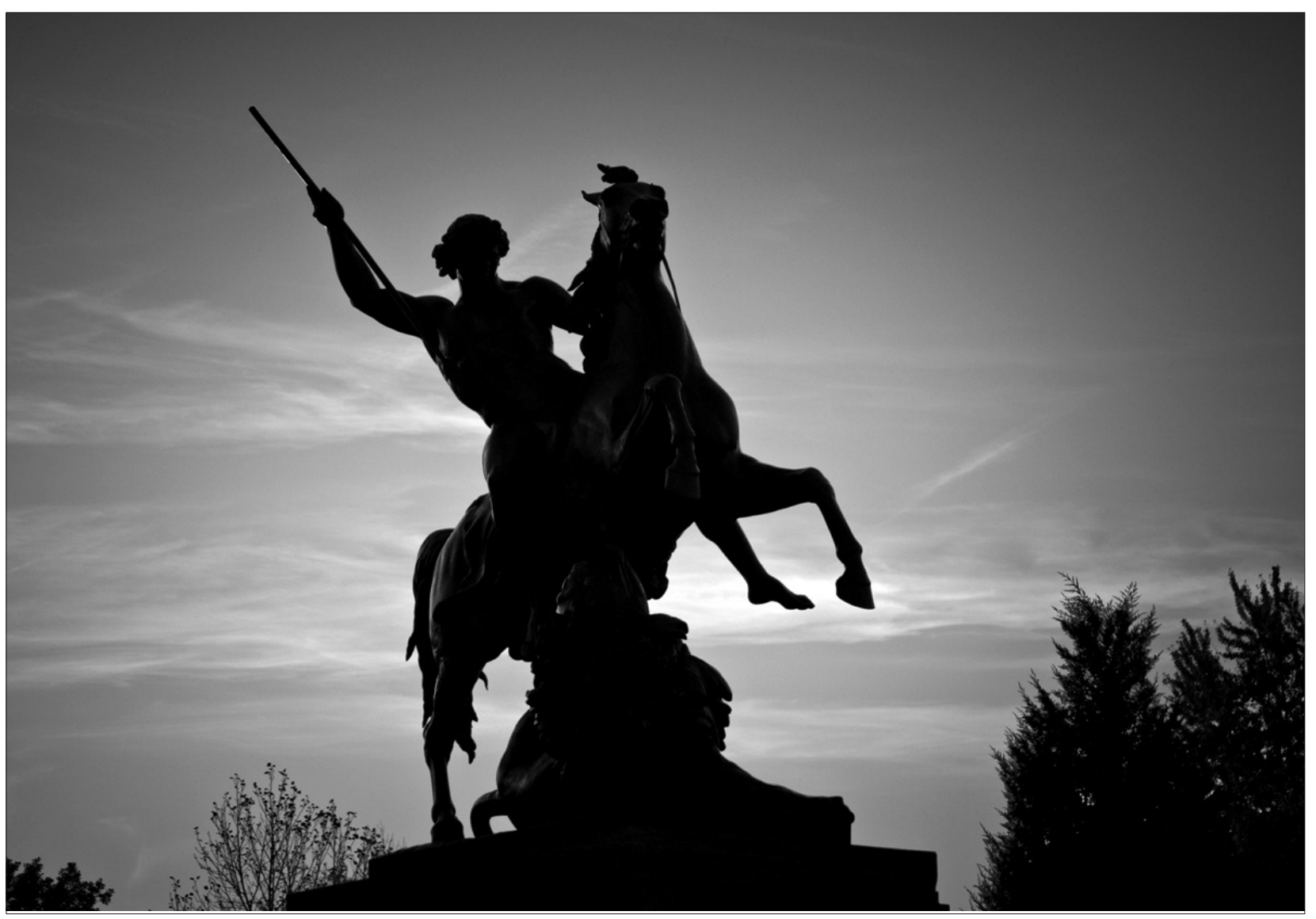

photograph by Soham Vakil, MD 